\title{
Product liftings and densities with lifting invariant and density invariant sections
}

\author{
by
}

\author{
K. Musiał (Wrocław), W. Straus s (Stuttgart) \\ and N. D. Macheras (Piraeus)
}

\begin{abstract}
Given two measure spaces equipped with liftings or densities (complete if liftings are considered) the existence of product liftings and densities with lifting invariant or density invariant sections is investigated. It is proved that if one of the marginal liftings is admissibly generated (a subclass of consistent liftings), then one can always find a product lifting which has the property that all sections determined by one of the marginal spaces are lifting invariant (Theorem 2.13). For a large class of measures Theorem 2.13 is the best possible (Theorem 4.3). When densities are considered, then one can always have a product density with measurable sections, but in the case of non-atomic complete marginal measures there exists no product density with all sections being density invariant. The results are then applied to stochastic processes.
\end{abstract}

Introduction. It follows from a result of Talagrand [13] that given a complete finite measure space $(\Omega, \Sigma, \mu)$ there exists a lifting (called consistent $) \varrho$ on $(\Omega, \Sigma, \mu)$ and a lifting $\pi$ on the direct product $(\Omega, \Sigma, \mu) \widehat{\otimes}(\Omega, \Sigma, \mu)$ satisfying the equality

$$
\pi\left(E_{1} \times E_{2}\right)=\varrho\left(E_{1}\right) \times \varrho\left(E_{2}\right)
$$

for arbitrary $E_{1}, E_{2} \in \Sigma$.

Macheras and Strauss [9] proved that given complete finite measure spaces $(\Omega, \Sigma, \mu),(\Theta, T, \nu)$ and a fixed lifting $\varrho$ on $(\Omega, \Sigma, \mu)$, one can find liftings $\sigma$ on $(\Theta, T, \nu)$ and $\pi$ on $(\Omega, \Sigma, \mu) \widehat{\otimes}(\Theta, T, \nu)$ such that $\pi \in \varrho \otimes \sigma$, i.e.

$$
\pi(A \times B)=\varrho(A) \times \sigma(B) \quad \text { for all } A \in \Sigma, B \in T .
$$

There is now a question whether it is possible to get a lifting $\pi$ that satisfies $(\mathrm{P})$ and has all sections of $\pi(E)$ lifting invariant for each $E \in \Sigma \widehat{\otimes} T$.

2000 Mathematics Subject Classification: Primary 28A51; Secondary 60A10, 60G05.

Research of N. D. Macheras partially supported by the Research Center of Piraeus University. 
We show that given measure spaces $(\Omega, \Sigma, \mu),(\Theta, T, \nu)$ and an arbitrary lifting $\varrho$ for $\mu$ there exist liftings $\sigma$ for $\nu$ and $\pi$ for $\mu \widehat{\otimes} \nu$ satisfying (P) and such that for each $E \in \Sigma \widehat{\otimes} T$ and each $\omega$, we have $[\pi(E)]_{\omega}=\sigma\left([\pi(E)]_{\omega}\right)$. In the case of identical measure spaces, a similar result holds true but for $\varrho$ taken from a special family of liftings, which we call "admissibly generated".

We also prove however that if $(\Omega, \Sigma, \mu)$ and $(\Theta, T, \nu)$ are non-atomic, then there exist no liftings $\varrho$ for $\mu, \sigma$ for $\nu$ and $\pi$ for $\mu \widehat{\otimes} \nu$ such that for each set $\pi(E)$ all sections $[\pi(E)]^{\theta}$ are invariant with respect to $\varrho$ and all sections $[\pi(E)]_{\omega}$ are invariant with respect to $\sigma$.

1. Preliminaries. Given a probability space $(\Omega, \Sigma, \mu)$ the family of all $\mu$-null sets is denoted by $\Sigma_{0}$. The (Carathéodory) completion of $(\Omega, \Sigma, \mu)$ will be denoted by $(\Omega, \widehat{\Sigma}, \widehat{\mu})$.

$\mathcal{L}^{1}(\mu)$ denotes the family of all real-valued $\mu$-integrable functions on $(\Omega, \Sigma, \mu)$; functions that are a.e. equal are not identified. $\mathcal{L}^{\infty}(\mu)$ denotes the family of all bounded real-valued $\mu$-measurable functions on $(\Omega, \Sigma, \mu)$; functions that are a.e. equal are not identified. The space of equivalence classes of members of $\mathcal{L}^{1}(\mu)$ and $\mathcal{L}^{\infty}(\mu)$ is denoted by $L^{1}(\mu)$ and $L^{\infty}(\mu)$, respectively. The $\sigma$-algebra generated by a family $\mathcal{L}$ of sets is denoted by $\sigma(\mathcal{L})$.

If $f \in \mathcal{L}^{1}(\mu)$ and $\eta$ is a sub- $\sigma$-algebra of $\Sigma$, then each function $g \in$ $\mathcal{L}^{1}(\mu \mid \eta)$ satisfying $\int_{A} f d \mu=\int_{A} g d \mu$ for each $A \in \eta$ is said to be a version of the conditional expectation of $f$ with respect to $\eta$. It will be denoted by $E_{\eta}(f)$.

$\mathbb{N}$ stands for the natural numbers. If $M \subseteq \Omega$, then $M^{\mathrm{c}}:=\Omega \backslash M$. We use the notion of lower density and lifting in the sense of [6] and, for any probability space $(\Omega, \Sigma, \mu)$ we denote by $\vartheta(\mu)$ and by $\Lambda(\mu)$ the systems of all lower densities and liftings, respectively. Since all densities considered in this paper are lower densities we will use the word "density" instead of "lower density".

We denote by $(\Omega \times \Theta, \Sigma \otimes T, \mu \otimes \nu)$ the product probability space of the probability spaces $(\Omega, \Sigma, \mu)$ and $(\Theta, T, \nu) .(\Omega \times \Theta, \Sigma \widehat{\otimes} T, \mu \widehat{\otimes} \nu)$ denotes its (Carathéodory) completion.

If $f$ is a function defined on $\Omega \times \Theta$ and $(\omega, \theta) \in \Omega \times \Theta$ is fixed, then we use the ordinary notation $f_{\omega}, f^{\theta}$ for the functions obtained from $f$ by fixing $\omega$ and $\theta$ respectively. In a similar way the sections $E_{\omega}, E^{\theta}$ of a set $E \subseteq \Omega \times \Theta$ are defined. A filter $\mathcal{F}$ in $\Sigma$ is said to be $\mu$-stable if $A \in \mathcal{F}$ and $\mu(A \triangle B)=0$ yields $B \in \mathcal{F}$.

2. Existence of densities and liftings with one-sided density invariant or lifting invariant sections. We start with the following general result: 
Lemma 2.1. Let $(\Omega, \Sigma, \mu)$ and $(\Theta, T, \nu)$ be probability spaces and let $\eta$ be a sub- $\sigma$-algebra of $T$. Then for each $f \in \mathcal{L}^{\infty}(\mu \otimes \nu)$,

$$
\Omega \backslash\left\{\omega \in \Omega:\left[E_{\Sigma \otimes \eta}(f)\right]_{\omega}=E_{\eta}\left(f_{\omega}\right) \text { a.e. }(\nu \mid \eta)\right\} \in \widehat{\Sigma}_{0} .
$$

Proof. Denote by $\Sigma \times T$ the product algebra generated by $\Sigma$ and $T$ and let

$$
\mathcal{C}:=\left\{C \in \Sigma \otimes T: \chi_{C} \text { satisfies the assertion of the lemma }\right\} .
$$

One can easily see that $\Sigma \times T \subset \mathcal{C}$. Applying now the standard methods of integration theory and the Monotone Convergence Theorem for conditional expectations (cf. [1], Th. 34.2) one can see first that $\mathcal{C}$ is a monotone class of sets (and so $\mathcal{C}=\Sigma \otimes T$ ) and then that the required result holds true.

It is worth noticing that the assertion of Lemma 2.1 is in general false if one takes $\Theta$-sections instead of $\Omega$-sections.

ExAmple 2.2. Let $\Omega=\Theta=[0,1)$, let $\Sigma$ denote the Lebesgue measurable subsets of $[0,1)$ and let $\eta$ be the algebra generated by $[0,1 / 2)$. If $f=\chi_{[0,1) \times[0,1 / 3)}$ and $g=E_{\Sigma \otimes \eta}(f)$ then one can easily check that

$$
\forall \theta \in[1 / 3,1 / 2) g^{\theta}=2 / 3 \text { a.e. whereas } \forall \theta \in[1 / 3,1 / 2) f^{\theta}=0 \text { a.e. }
$$

It has already been observed by Talagrand [13] that not all liftings have good properties from the product point of view. The same holds true in our investigations concerning liftings and densities. Therefore we now isolate a wide class of densities with properties suitable for our purposes.

First, however, for completeness and because of the lack of one assumption in [5], we recall Lemma 2 of [5].

LemMa 2.3. Let $(\Theta, T, \nu)$ be a probability space and let $\eta$ be a sub- $\sigma$ algebra of $T$ such that $T_{0} \subset \eta$. Moreover, let $\delta \in \vartheta(\nu \mid \eta)$ and $M \in T \backslash \eta$. If $M_{1} \supset M$ and $M_{2} \supset M^{\mathrm{c}}$ are $\eta$-envelopes of $M$ and $M^{\mathrm{c}}$ respectively, then the formula

$$
\begin{aligned}
& \widetilde{\delta}\left[(G \cap M) \cup\left(H \cap M^{\mathrm{c}}\right)\right] \\
& \quad:=\left[M \cap \delta\left(\left(G \cap M_{1}\right) \cup\left(H \cap M_{1}^{\mathrm{c}}\right)\right)\right] \cup\left[M^{\mathrm{c}} \cap \delta\left(\left(H \cap M_{2}\right) \cup\left(G \cap M_{2}^{\mathrm{c}}\right)\right)\right]
\end{aligned}
$$

defines a density $\widetilde{\delta} \in \vartheta(\nu \mid \sigma(\eta \cup\{M\}))$ that is an extension of $\delta$.

Definition 2.4. Let $(\Theta, T, \nu)$ be a probability space. A density $\tau \in \vartheta(\nu)$ is called an admissible density if it can be constructed by transfinite induction as described below.

(A) Let $\mathcal{D}$ be the smallest cardinal with the property that there exists a collection $\mathcal{M} \subset T$ such that $\sigma(\mathcal{M})$ is dense in $T$ in the pseudometric generated by $\nu$. Let $\mathcal{M}=\left(M_{\alpha}\right)_{\alpha<\kappa}$ be numbered by the ordinals less than $\kappa$, where $\kappa$ is the first ordinal of cardinality $\mathcal{D}$. Denote by $\eta_{0}$ the $\sigma$ algebra $\sigma\left(T_{0}\right)$ and for each $1 \leq \alpha<\kappa$ let $\eta_{\alpha}$ be the $\sigma$-algebra generated by 
$\left\{M_{\gamma}: \gamma<\alpha\right\} \cup \eta_{0}$. We assume without loss of generality that $M_{\alpha} \notin \eta_{\alpha}$ for each $\alpha$.

(B) $\tau_{0} \in \vartheta\left(\nu \mid \eta_{0}\right)$ is the unique density existing on $\left(\Theta, \eta_{0}, \nu \mid \eta_{0}\right)$.

(C) If $\gamma<\kappa$ is a limit ordinal of uncountable cofinality, then $\eta_{\gamma}=$ $\bigcup_{\alpha<\gamma} \eta_{\alpha}$ and we define $\tau_{\gamma} \in \vartheta\left(\nu \mid \eta_{\gamma}\right)$ by setting

$$
\tau_{\gamma}(B):=\tau_{\alpha}(B) \quad \text { if } B \in \eta_{\alpha} \text { and } \alpha<\gamma .
$$

(D) Assume now that there exists an increasing sequence $\left(\gamma_{n}\right)$ of ordinals that is cofinal to $\gamma$. For simplicity put $\tau_{n}:=\tau_{\gamma_{n}}$ and $\eta_{n}:=\eta_{\gamma_{n}}$ for all $n \in \mathbb{N}$. Then $\eta_{\gamma}=\sigma\left(\bigcup_{n \in \mathbb{N}} \eta_{n}\right)$ and we can define $\tau_{\gamma}$ by setting

$$
\tau_{\gamma}(B):=\bigcap_{k \in \mathbb{N}} \bigcup_{n \in \mathbb{N}} \bigcap_{m \geq n} \tau_{m}\left(\left\{E_{\eta_{m}}\left(\chi_{B}\right)>1-1 / k\right\}\right) \quad \text { for } B \in \eta_{\gamma} .
$$

It follows by [5], Lemma 1 (or by Lemma 2.3), that $\tau_{\gamma} \in \vartheta\left(\nu \mid \eta_{\gamma}\right)$ and $\tau_{\gamma} \mid \eta_{n}=$ $\tau_{n}$ for each $n \in \mathbb{N}$.

(E) Let now $\gamma=\beta+1$. To simplify the notations let $M:=M_{\beta}$. It is well known that

$$
\eta_{\gamma}=\left\{(G \cap M) \cup\left(H \cap M^{\mathrm{c}}\right): G, H \in \eta_{\beta}\right\} .
$$

Let $M_{1} \supseteq M$ and $M_{2} \supseteq M^{\mathrm{c}}$ be $\eta_{\beta^{-}}$envelopes of $M$ and $M^{\mathrm{c}}$ respectively, i.e. $M_{1}, M_{2} \in \eta_{\beta},\left(\nu \mid \eta_{\beta}\right)_{*}\left(M_{1} \backslash M\right)=0$ and $\left(\nu \mid \eta_{\beta}\right)_{*}\left(M_{2} \backslash M^{\mathrm{c}}\right)=0$ (where $\left(\nu \mid \eta_{\beta}\right)_{*}$ is the inner measure induced by $\left.\nu \mid \eta_{\beta}\right)$. Define

$$
\begin{aligned}
& \tau_{\gamma}\left((G \cap M) \cup\left(H \cap M^{\mathrm{c}}\right)\right) \\
& \quad:=\left(M \cap \tau_{\beta}\left(\left(G \cap M_{1}\right) \cup\left(H \cap M_{1}^{\mathrm{c}}\right)\right)\right) \cup\left(M^{\mathrm{c}} \cap \tau_{\beta}\left(\left(H \cap M_{2}\right) \cup\left(G \cap M_{2}^{\mathrm{c}}\right)\right)\right)
\end{aligned}
$$

for $G, H \in \eta_{\beta}$. By [5], Lemma 2, it then follows that $\tau_{\gamma} \in \vartheta\left(\nu \mid \eta_{\gamma}\right)$ and $\tau_{\gamma} \mid \eta_{\beta}=\tau_{\beta}$

(F) We define $\tau \in \vartheta(\nu)$ by setting $\tau=\tau_{\kappa}$.

Throughout, the collection of all admissible densities on $(\Theta, T, \nu)$ will be denoted by $A \vartheta(\nu)$ and each $\tau \in A \vartheta(\nu)$ will be considered together with all elements involved in the above construction without any additional remarks.

THeOREM 2.5. Let $(\Theta, T, \nu)$ be an arbitrary probability space. If $\tau \in$ $A \vartheta(\nu)$ then for each $(\Omega, \Sigma, \mu)$ and each $\delta \in \vartheta(\mu)$ there exists $\varphi_{1} \in \vartheta(\mu \otimes \nu)$ such that $\varphi_{1} \in \delta \otimes \tau$ and for every $E \in \Sigma \otimes T$ there exists $N_{E} \in \Sigma_{0}$ with

$$
\left[\varphi_{1}(E)\right]_{\omega}=\tau\left(\left[\varphi_{1}(E)\right]_{\omega}\right) \quad \text { for all } \omega \in \Omega \backslash N_{E} .
$$

Proof. Let there be given a $\delta \in \vartheta(\mu)$ and $\tau \in A \vartheta(\nu)$ together with other elements involved in the construction of $\tau \in A \vartheta(\nu)$. In particular the family $\mathcal{M}=\left(M_{\alpha}\right)_{\alpha<\kappa}$, the $\sigma$-subalgebras $\left(\eta_{\alpha}\right)_{\alpha<\kappa}$ and the sequences $\left(\gamma_{n}\right)$ cofinal with limit ordinals are fixed.

Using transfinite induction, we now construct a transfinite sequence $\left(\varphi_{\alpha}\right)_{\alpha \leq \kappa}$ with $\varphi_{\alpha} \in \vartheta\left(\mu \otimes \nu \mid \Sigma \otimes \eta_{\alpha}\right)$ such that: 
- for all $A \in \Sigma, B \in \eta_{\alpha}$,

$$
\varphi_{\alpha}(A \times B)=\delta(A) \times \tau_{\alpha}(B)
$$

- for all $\alpha \leq \gamma \leq \kappa$,

$$
\varphi_{\gamma} \mid \Sigma \otimes \eta_{\alpha}=\varphi_{\alpha}
$$

- for each $\alpha \leq \kappa$ and each $E \in \Sigma \otimes \eta_{\alpha}$ there exists $N_{E} \in \Sigma_{0}$ with

$$
\left[\varphi_{\alpha}(E)\right]_{\omega}=\tau_{\alpha}\left(\left[\varphi_{\alpha}(E)\right]_{\omega}\right) \quad \text { for all } \omega \in \Omega \backslash N_{E} .
$$

For the first step, if $E \in \Sigma \otimes \eta_{0}$ then $E=\widetilde{E} \times \Theta$ a.e. $(\mu \otimes \nu)$ for some $\widetilde{E} \in \Sigma$. Hence, defining

$$
\varphi_{0}(E)=\delta(\widetilde{E}) \times \Theta \quad \text { for all } E=\widetilde{E} \times \Theta \text { a.e. }(\mu \otimes \nu)
$$

we get $\left.\varphi_{0} \in \vartheta(\mu \otimes \nu) \mid \Sigma \otimes \eta_{0}\right)$ satisfying (1)-(3).

Assume now, that given $1 \leq \gamma<\kappa$, a system $\left(\varphi_{\alpha}\right)$ satisfying (1)-(3) has been constructed for all $\alpha<\gamma$. We have to distinguish three cases.

CASE A: $\gamma$ is a limit ordinal of uncountable cofinality. Then $\Sigma \otimes \eta_{\gamma}=$ $\bigcup_{\alpha<\gamma}\left(\Sigma \otimes \eta_{\alpha}\right)$. Setting

$$
\varphi_{\gamma}(E)=\varphi_{\alpha}(E) \quad \text { if } E \in \Sigma \otimes \eta_{\alpha},
$$

we get a uniquely defined density $\varphi_{\gamma} \in \vartheta\left(\mu \otimes \nu \mid \Sigma \otimes \eta_{\gamma}\right)$ satisfying (1)-(3).

CASE B: $\gamma$ is of countable cofinality. For simplicity put $\tau_{n}:=\tau_{\gamma_{n}}, \varphi_{n}:=$ $\varphi_{\gamma_{n}}$ and $\eta_{n}:=\eta_{\gamma_{n}}$ for all $n \in \mathbb{N}$. Then

$$
\Sigma \otimes \eta_{\gamma}=\sigma\left(\bigcup_{n \in \mathbb{N}} \Sigma \otimes \eta_{n}\right)
$$

Hence, we can define

$$
\varphi_{\gamma}(P):=\bigcap_{k \in \mathbb{N}} \bigcup_{n \in \mathbb{N}} \bigcap_{m \geq n} \varphi_{m}\left(\left\{E_{\Sigma \otimes \eta_{m}}\left(\chi_{P}\right)>1-1 / k\right\}\right) \quad \text { for } P \in \Sigma \otimes \eta_{\gamma} .
$$

It follows by [5], Lemma 1, that $\varphi_{\gamma} \in \vartheta\left(\mu \otimes \nu \mid \Sigma \otimes \eta_{\gamma}\right)$, and $\varphi_{\gamma} \mid \Sigma \otimes \eta_{n}=\varphi_{n}$ for each $n \in \mathbb{N}$. Moreover, by [9] (Section 2, proof of Theorem 3) we have $\varphi_{\gamma} \in \delta \otimes \tau_{\gamma}$.

For $P \in \Sigma \otimes \eta_{\gamma}$, fix for each $m \in \mathbb{N}$ a version $E_{\Sigma \otimes \eta_{m}}\left(\chi_{P}\right)$ of conditional expectation. Then, by (3), for all $k, m \in \mathbb{N}$ there exists $N_{m, k} \in \Sigma_{0}$ such that

$$
\left[\varphi_{m}\left(\left\{E_{\Sigma \otimes \eta_{m}}\left(\chi_{P}\right)>1-1 / k\right\}\right)\right]_{\omega}=\tau_{m}\left(\left\{E_{\Sigma \otimes \eta_{m}}\left(\chi_{P}\right)>1-1 / k\right\}_{\omega}\right)
$$

if $\omega \in \Omega \backslash N_{m, k}$.

By Lemma 2.1 there exist $N_{m} \in \Sigma_{0}$ with

$$
\left[E_{\Sigma \otimes \eta_{m}}\left(\chi_{P}\right)\right]_{\omega}=E_{\eta_{m}}\left(\chi_{P_{\omega}}\right) \quad \text { a.e. }(\nu) \text { for all } \omega \in \Omega \backslash N_{m} \text {. }
$$


So if we put $N_{P}:=\bigcup_{k, m \in \mathbb{N}}\left(N_{m, k} \cup N_{m}\right)$ and take into account the equality

$$
\left\{E_{\Sigma \otimes \eta_{m}}\left(\chi_{P}\right)>1-1 / k\right\}_{\omega}=\left\{\left[E_{\Sigma \otimes \eta_{m}}\left(\chi_{P}\right)\right]_{\omega}>1-1 / k\right\},
$$

which holds true for arbitrary $\omega \in \Omega$, then we have $N_{P} \in \Sigma_{0}$ and

$$
\begin{aligned}
{\left[\varphi_{\gamma}(P)\right]_{\omega} } & =\bigcap_{k \in \mathbb{N}} \bigcup_{n \in \mathbb{N}} \bigcap_{m \geq n}\left[\varphi_{m}\left(\left\{E_{\Sigma \otimes \eta_{m}}\left(\chi_{P}\right)>1-1 / k\right\}\right)\right]_{\omega} \\
& =\bigcap_{k \in \mathbb{N}} \bigcup_{n \in \mathbb{N}} \bigcap_{m \geq n} \tau_{m}\left(\left\{E_{\eta_{m}}\left(\chi_{P_{\omega}}\right)>1-1 / k\right\}\right)=\tau_{\gamma}\left(P_{\omega}\right)
\end{aligned}
$$

for $\omega \in \Omega \backslash N_{P}$. Hence (3) is satisfied.

CASE C: $\gamma=\beta+1$. To simplify the notations let $M:=M_{\beta}$. It is well known that

$$
\Sigma \otimes \eta_{\gamma}=\left\{(K \cap(\Omega \times M)) \cup\left(L \cap\left(\Omega \times M^{\mathrm{c}}\right)\right): K, L \in \Sigma \otimes \eta_{\beta}\right\} .
$$

Let $M_{1} \supseteq M$ and $M_{2} \supseteq M^{\mathrm{c}}$ be $\eta_{\beta}$-envelopes of $M$ and $M^{\mathrm{c}}$ respectively, used in the process of describing $\tau_{\gamma}$.

An easy calculation shows that

$$
E_{1}=\Omega \times M_{1} \quad \text { and } \quad E_{2}=\Omega \times M_{2}
$$

are $\Sigma \otimes \eta_{\beta^{-}}$-envelopes of $\Omega \times M$ and $\Omega \times M^{\mathrm{c}}$, respectively. Define

$$
\begin{aligned}
\varphi_{\gamma}((K \cap(\Omega \times M)) \cup(L \cap & \left.\left.\left(\Omega \times M^{\mathrm{c}}\right)\right)\right) \\
:= & \left((\Omega \times M) \cap \varphi_{\beta}\left(\left(K \cap E_{1}\right) \cup\left(L \cap E_{1}^{\mathrm{c}}\right)\right)\right) \\
& \cup\left(\left(\Omega \times M^{\mathrm{c}}\right) \cap \varphi_{\beta}\left(\left(L \cap E_{2}\right) \cup\left(K \cap E_{2}^{\mathrm{c}}\right)\right)\right)
\end{aligned}
$$

for $K, L \in \Sigma \otimes \eta_{\beta}$. By [5], Lemma 2, it then follows that $\varphi_{\gamma} \in \vartheta\left(\mu \otimes \nu \mid \Sigma \otimes \eta_{\gamma}\right)$, $\varphi_{\gamma} \mid \Sigma \otimes \eta_{\beta}=\varphi_{\beta}$, and

$$
\varphi_{\gamma}(A \times B)=\delta(A) \times \tau_{\gamma}(B) \quad \text { for all } A \in \Sigma \text { and } B \in \eta_{\gamma},
$$

that is, $\varphi_{\gamma}$ satisfies (1) and (2). For $E \in \Sigma \otimes \eta_{\gamma}$ write

$$
E=(K \cap(\Omega \times M)) \cup\left(L \cap\left(\Omega \times M^{\mathrm{c}}\right)\right) \quad \text { with } K, L \in \Sigma \otimes \eta_{\beta}
$$

and put

$$
M_{E}:=N_{\left(K \cap E_{1}\right) \cup\left(L \cap E_{1}^{c}\right)} \cup N_{\left(L \cap E_{2}\right) \cup\left(K \cap E_{2}^{\mathrm{c}}\right)},
$$

where $N_{\left(K \cap E_{1}\right) \cup\left(L \cap E_{1}^{c}\right)}$ and $N_{\left(L \cap E_{2}\right) \cup\left(K \cap E_{2}^{c}\right)}$ are taken from (3) with $\alpha=\beta$. Moreover, let $K_{E} \in \Sigma_{0}$ be such that for $\omega \notin K_{E}$ the sets $\left(\varphi_{\beta}\left[\left(K \cap E_{1}\right)\right.\right.$ $\left.\left.\cup\left(L \cap E_{1}^{c}\right)\right]\right)_{\omega}$ and $\left[\left(K \cap E_{1}\right) \cup\left(L \cap E_{1}^{c}\right)\right]_{\omega}$ as well as the sets $\left(\varphi_{\beta}\left[\left(L \cap E_{2}\right) \cup\right.\right.$ $\left.\left.\left(K \cap E_{2}^{\mathrm{c}}\right)\right]\right)_{\omega}$ and $\left[\left(L \cap E_{2}\right) \cup\left(K \cap E_{2}^{\mathrm{c}}\right)\right]_{\omega}$ are $\nu$-equivalent. Then $N_{E}:=$ $M_{E} \cup K_{E} \in \Sigma_{0}$ and $\left[\varphi_{\gamma}(E)\right]_{\omega}=\tau_{\gamma}\left(E_{\omega}\right)$ for $\omega \in \Omega \backslash N_{E}$. Therefore $\varphi_{\gamma}$ satisfies the conditions (1)-(3).

We can now define $\varphi \in \vartheta(\mu \otimes \nu)$ with the required properties by just setting $\varphi=\varphi_{\kappa}$. The densities are properly defined, since each element of $\Sigma \otimes T$ is measurable with respect to some $\Sigma \otimes \eta_{\alpha}$ with $\alpha \leq \kappa$. 
Remark 2.6. Some of our results will be applied in proofs in two forms. More precisely, the role of measure spaces $(\Omega, \Sigma, \mu)$ and $(\Theta, T, \nu)$ is often interchanged without special comments. We shall denote it by putting the symbol ${ }^{\perp}$ after the number of a relevant theorem.

Corollary 2.7. Let $(\Theta, T, \nu)$ be an arbitrary probability space. If $\tau \in$ $A \vartheta(\nu)$ then for each $(\Omega, \Sigma, \mu)$ and each $\delta \in \vartheta(\mu)$ there exists $\bar{\varphi}_{1} \in \vartheta(\mu \widehat{\otimes} \nu)$ with the following properties:

(i) $\bar{\varphi}_{1}(A \times B)=\delta(A) \times \tau(B)$ for all $A \in \Sigma$ and $B \in T$;

(ii) $\left[\bar{\varphi}_{1}(E)\right]_{\omega}=\tau\left(\left[\bar{\varphi}_{1}(E)\right]_{\omega}\right)$ for all $\omega \in \Omega$ and $E \in \Sigma \widehat{\otimes} T$;

(iii) $\left[\bar{\varphi}_{1}(E)\right]^{\theta}$ is $\widehat{\mu}$-measurable for all $\theta \in \Theta$ and $E \in \Sigma \widehat{\otimes} T$.

Proof. If $\delta \in \vartheta(\mu)$ and $\tau \in A \vartheta(\nu)$ then, according to Theorem 2.5, there exists $\varphi_{1} \in \delta \otimes \tau$ satisfying for each $E \in \Sigma \otimes T$ the equality $\left[\varphi_{1}(E)\right]_{\omega}=$ $\tau\left(\left[\varphi_{1}(E)\right]_{\omega}\right)$ for almost all $\omega$.

Let $E \in \Sigma \otimes T$ be an arbitrary set. We define $\bar{\varphi}_{1}(E)$ by setting

$$
\left[\bar{\varphi}_{1}(E)\right]_{\omega}=\tau\left(\left[\varphi_{1}(E)\right]_{\omega}\right) \quad \text { for all } \omega \in \Omega .
$$

It can be easily seen that $\bar{\varphi}_{1}$ satisfies the required conditions.

Lemma 2.8. Let $(\Omega, \Sigma, \mu)$ and $(\Theta, T, \nu)$ be complete. Moreover, let $\tau \in \vartheta(\nu)$ be an arbitrary density and $\xi \in \vartheta(\mu \widehat{\otimes} \nu)$ be a density such that for each $(\omega, \theta) \in \Omega \times \Theta$ and $E \in \Sigma \widehat{\otimes} T$ we have $[\xi(E)]_{\omega}=\tau\left([\xi(E)]_{\omega}\right)$ and the set $[\xi(E)]^{\theta}$ is $\mu$-measurable. Then there exists $\psi_{1} \in \vartheta(\mu \widehat{\otimes} \nu)$ satisfying for each $(\omega, \theta) \in \Omega \times \Theta$ and $E \in \Sigma \widehat{\otimes} T$ the following conditions:

(j) $\xi(E) \subseteq \psi_{1}(E)$;

(jj) $\nu\left(\left[\psi_{1}(E)\right]_{\omega} \cup\left[\psi_{1}\left(E^{\mathrm{c}}\right)\right]_{\omega}\right)=1$;

(jjj) $\left[\psi_{1}(E)\right]_{\omega}=\tau\left(\left[\psi_{1}(E)\right]_{\omega}\right)$;

(jv) $\left[\psi_{1}(E)\right]^{\theta}$ is measurable.

If additionally $\xi$ is such that $[\xi(A \times \Theta)]_{\omega} \in\{\emptyset, \Theta\}$ for each $\omega \in \Omega$ and $A \in \Sigma$, then one can choose $\psi_{1}$ in such a way that for each $\omega \in \Omega$ and each $A \in \Sigma$,

(v) $\left[\psi_{1}(A \times \Theta)\right]_{\omega} \in\{\emptyset, \Theta\}$ and $\left[\psi_{1}(A \times \Theta)\right]_{\omega} \cup\left[\psi_{1}\left(A^{\mathrm{c}} \times \Theta\right)\right]_{\omega}=\Theta$.

Proof. Let

$\Phi:=\left\{\varphi \in \vartheta(\mu \widehat{\otimes} \nu): \forall \omega \in \Omega \forall E \in \Sigma \widehat{\otimes} T[\varphi(E)]_{\omega} \subseteq \tau\left([\varphi(E)]_{\omega}\right)\right.$

$\left.\& \forall E \in \Sigma \widehat{\otimes} T \xi(E) \subseteq \varphi(E) \& \forall A \in \Sigma \forall \omega \in \Omega[\varphi(A \times \Theta)]_{\omega} \in\{\emptyset, \Theta\}\right\}$.

Notice first that $\Phi \neq \emptyset$ since $\xi \in \Phi$.

We consider $\Phi$ with inclusion as partial order: $\varphi \leq \widetilde{\varphi}$ if $\varphi(E) \subseteq \widetilde{\varphi}(E)$ for each $E \in \Sigma \widehat{\otimes} T$.

Claim 1. There exists a maximal element in $\Phi$. 
Pro of. We only have to prove that each chain $\left\{\varphi_{\alpha}\right\}_{\alpha \in A} \subseteq \Phi$ has a dominating element in $\Phi$. The obvious candidate is $\varphi$ given for each $E \in$ $\Sigma \widehat{\otimes} T$ by

$$
\varphi(E)=\bigcup_{\alpha \in A} \varphi_{\alpha}(E)
$$

One can easily prove that $\varphi \in \vartheta(\mu \widehat{\otimes} \nu)$.

Consider now the section properties of $\varphi(E)$. For fixed $\omega \in \Omega$,

$$
[\varphi(E)]_{\omega}=\bigcup_{\alpha \in A}\left[\varphi_{\alpha}(E)\right]_{\omega} \subseteq \bigcup_{\alpha \in A} \tau\left(\left[\varphi_{\alpha}(E)\right]_{\omega}\right)
$$

and so (in virtue of [6], Chapter III, Section 3) the set $[\varphi(E)]_{\omega}$ is measurable. It is clear that also $[\varphi(E)]_{\omega} \subseteq \tau\left([\varphi(E)]_{\omega}\right)$. This proves that $\varphi$ dominates the whole chain. According to the Zorn-Kuratowski Lemma the set $\Phi$ has a maximal element $\psi_{1}$.

Claim 2. For each $\omega \in \Omega$ and $A \in \Sigma$,

$$
\left[\psi_{1}(A \times \Theta)\right]_{\omega} \cup\left[\psi_{1}\left(A^{\mathrm{c}} \times \Theta\right)\right]_{\omega}=\Theta .
$$

P r o o f. According to the definition of $\Phi$ we have $\left[\psi_{1}(A \times \Theta)\right]_{\omega} \in\{\emptyset, \Theta\}$ for each $\omega$ and $A \in \Sigma$. Suppose that $\left[\psi_{1}\left(A_{0} \times \Theta\right)\right]_{\omega} \cup\left[\psi_{1}\left(A_{0}^{\mathrm{c}} \times \Theta\right)\right]_{\omega}=\emptyset$ for some $\omega_{0}$ and $A_{0} \in \Sigma$. Then define $\widehat{\psi} \in \vartheta(\mu \widehat{\otimes} \nu)$ by

$$
[\widehat{\psi}(E)]_{\omega}= \begin{cases}{\left[\psi_{1}(E)\right]_{\omega}} & \text { if } \omega \neq \omega_{0} \\ {\left[\psi_{1}\left(E \cup\left(A_{0} \times \Theta\right)\right)\right]_{\omega_{0}}} & \text { if } \omega=\omega_{0} .\end{cases}
$$

It is clear that $\psi_{1}(E) \subseteq \widehat{\psi}(E)$ for each $E \in \Sigma \widehat{\otimes} T$ and $[\widehat{\psi}(A \times \Theta)]_{\omega} \in\{\emptyset, \Theta\}$ for each $\omega \in \Omega$ and $A \in \Sigma$. Since $\left[\widehat{\psi}\left(A_{0}^{\mathrm{c}} \times \Theta\right)\right]_{\omega_{0}}=\Theta$ we have $\psi_{1} \neq \widehat{\psi}$, which contradicts the maximality of $\psi_{1}$.

Claim 3. For each $\omega \in \Omega$ and $E \in \Sigma \otimes \Theta$,

$$
\nu\left(\left[\psi_{1}(E)\right]_{\omega} \cup\left[\psi_{1}\left(E^{\mathrm{c}}\right)\right]_{\omega}\right)=1 .
$$

Proof. If not, then there exist $H \in \Sigma \widehat{\otimes} T$ and $\omega_{0} \in \Omega$ such that $\nu\left(\left[\psi_{1}(H)\right]_{\omega_{0}} \cup\left[\psi_{1}\left(H^{\mathrm{c}}\right)\right]_{\omega_{0}}\right)<1$. Let

$$
W:=\tau\left[\left(\left[\psi_{1}(H)\right]_{\omega_{0}} \cup\left[\psi_{1}\left(H^{\mathrm{c}}\right)\right]_{\omega_{0}}\right)^{\mathrm{c}}\right]
$$

and let

$$
[\widehat{\psi}(E)]_{\omega}= \begin{cases}{\left[\psi_{1}(E)\right]_{\omega}} & \text { if } \omega \neq \omega_{0} \\ {\left[\psi_{1}(E)\right]_{\omega_{0}} \cup\left(W \cap\left[\psi_{1}(H \cup E)\right]_{\omega_{0}}\right)} & \text { if } \omega=\omega_{0} .\end{cases}
$$

It is clear that $\psi_{1}(E) \subseteq \widehat{\psi}(E)$ for each $E \in \Sigma \widehat{\otimes} T$. One easily checks that $\widehat{\psi} \in \vartheta(\mu \widehat{\otimes} \nu)$. 
It follows directly from the definition that $\widehat{\psi}$ and $\psi_{1}$ are different densities. To get a contradiction it is enough to show that $[\widehat{\psi}(E)]_{\omega_{0}} \subseteq \tau\left([\widehat{\psi}(E)]_{\omega_{0}}\right)$ and $[\widehat{\psi}(A \times \Theta)]_{\omega_{0}} \in\{\emptyset, \Theta\}$. If $E \in \Sigma \widehat{\otimes} T$, then

$$
\begin{aligned}
\tau\left([\widehat{\psi}(E)]_{\omega_{0}}\right) & \supseteq \tau\left(\left[\psi_{1}(E)\right]_{\omega_{0}}\right) \cup \tau\left(W \cap\left[\psi_{1}(H \cup E)\right]_{\omega_{0}}\right) \\
& \supseteq\left[\psi_{1}(E)\right]_{\omega_{0}} \cup\left[\tau(W) \cap \tau\left(\left[\psi_{1}(H \cup E)\right]_{\omega_{0}}\right)\right] \\
& \supseteq\left[\psi_{1}(E)\right]_{\omega_{0}} \cup\left(W \cap\left[\psi_{1}(H \cup E)\right]_{\omega_{0}}\right)=[\widehat{\psi}(E)]_{\omega_{0}} .
\end{aligned}
$$

One can also easily check that if $A \in \Sigma$, then $[\widehat{\psi}(A \times \Theta)]_{\omega_{0}} \in\{\emptyset, \Theta\}$.

In order to finish the proof of Lemma 2.8, we should still prove the validity of (jjj) and (v). For each $\omega \in \Omega$ and $E \in \Sigma \widehat{\otimes} T$ set

$$
[\widetilde{\psi}(E)]_{\omega}=\tau\left(\left[\psi_{1}(E)\right]_{\omega}\right) .
$$

Clearly $\psi_{1}(F) \subseteq \widetilde{\psi}(F)$ for each $F$. Moreover the equality $\psi_{1}(E) \cap \psi_{1}\left(E^{\mathrm{c}}\right)=$ $\emptyset$ yields $\tau\left(\left[\psi_{1}(E)\right]_{\omega}\right) \cap \tau\left(\left[\psi_{1}\left(E^{\mathrm{c}}\right)\right]_{\omega}\right)=\emptyset$ for each $\omega$. As a consequence, $\widetilde{\psi}\left(E^{\mathrm{c}}\right) \subseteq(\widetilde{\psi}(E))^{\mathrm{c}}$. Hence

$$
\psi_{1}\left(E^{\mathrm{c}}\right) \subseteq \widetilde{\psi}\left(E^{\mathrm{c}}\right) \subseteq[\widetilde{\psi}(E)]^{\mathrm{c}} \subseteq\left[\psi_{1}(E)\right]^{\mathrm{c}}
$$

and so the measurability of $\widetilde{\psi}(E)$ is proven. It follows that $\widetilde{\psi} \in \Phi$ and $\psi_{1}=$ $\widetilde{\psi}$. In order to prove the measurability of the $\Theta$-sections of $\psi_{1}$ notice that $(\mu \widehat{\otimes} \nu)\left(\xi(E) \triangle \psi_{1}(E)\right)=0$ since $\xi$ and $\psi_{1}$ are densities in the same measure space. It then follows from the Fubini Theorem that there is $M_{E} \in \Sigma_{0}$ such that for all $\omega \notin M_{E}$,

$$
\nu\left([\xi(E)]_{\omega} \triangle\left[\psi_{1}(E)\right]_{\omega}\right)=0 \quad \text { and } \quad \nu\left([\xi(E)]_{\omega} \cup\left[\xi\left(E^{\mathrm{c}}\right)\right]_{\omega}\right)=1 .
$$

Hence

$$
\xi(E) \backslash\left(M_{E} \times \Theta\right)=\psi_{1}(E) \backslash\left(M_{E} \times \Theta\right) .
$$

Since all sections $[\xi(E)]^{\theta}$ are measurable, the same holds true for the sections $\left[\psi_{1}(E)\right]^{\theta}$. This completes the proof of Lemma 2.8.

Theorem 2.9. Let $(\Theta, T, \nu)$ be complete. Then for each $\tau \in A \vartheta(\nu)$, each complete $(\Omega, \Sigma, \mu)$ and each $\delta \in \vartheta(\mu)$ there exist $\psi_{1} \in \vartheta(\mu \widehat{\otimes} \nu)$ and $\widetilde{\delta} \in \Lambda(\mu)$ satisfying the following conditions:

(i) $\delta(A) \times \tau(B) \subseteq \widetilde{\delta}(A) \times \tau(B)=\psi_{1}(A \times B)$ for all $A \in \Sigma$ and $B \in T$;

(ii) $\nu\left(\left[\psi_{1}(E)\right]_{\omega} \cup\left[\psi_{1}\left(E^{\mathrm{c}}\right)\right]_{\omega}\right)=1$ for all $\omega \in \Omega$ and $E \in \Sigma \widehat{\otimes} T$;

(iii) $\left[\psi_{1}(E)\right]_{\omega}=\tau\left(\left[\psi_{1}(E)\right]_{\omega}\right)$ for all $\omega \in \Omega$ and $E \in \Sigma \widehat{\otimes} T$;

(iv) $\left[\psi_{1}(E)\right]^{\theta}$ is measurable for all $\theta \in \Theta$ and $E \in \Sigma \widehat{\otimes} T$.

Proof. If $\delta \in \vartheta(\mu)$ and $\tau \in A \vartheta(\nu)$ are given, then there exists $\bar{\varphi}_{1} \in$ $\vartheta(\mu \widehat{\otimes} \nu)$ satisfying the conditions (i)-(iii) of Corollary 2.7. Let

$$
\begin{array}{r}
\Phi:=\left\{\varphi \in \vartheta(\mu \widehat{\otimes} \nu): \forall \omega \in \Omega \forall E \in \Sigma \widehat{\otimes} T[\varphi(E)]_{\omega} \subseteq \tau\left([\varphi(E)]_{\omega}\right)\right. \\
\left.\& \forall E \in \Sigma \widehat{\otimes} T \bar{\varphi}_{1}(E) \subseteq \varphi(E)\right\} .
\end{array}
$$


Notice first that $\Phi \neq \emptyset$ since $\bar{\varphi}_{1} \in \Phi$. According to Lemma 2.8 there exists $\psi_{1} \in \vartheta(\mu \widehat{\otimes} \nu)$ satisfying the conditions (jj)-(v) of Lemma 2.8 and the inclusion $\bar{\varphi}_{1}(E) \subseteq \psi_{1}(E)$. Since $\bar{\varphi}_{1}(A \times B)=\delta(A) \times \tau(B)$ for all $A \in \Sigma$ and $B \in T$, the inclusion $\delta(A) \times \tau(B) \subseteq \psi_{1}(A \times B)$ of (i) is satisfied. Thus, setting $\widetilde{\delta}(A):=\left\{\omega:\left[\psi_{1}(A \times \Theta)\right]_{\omega}=\Theta\right\}$ for each $A \in \Sigma$, we get $\widetilde{\delta} \in \Lambda(\mu)$. It follows that $\widetilde{\delta}(A) \times \Theta=\psi_{1}(A \times \Theta)$.

We now prove that $\psi_{1}(\Omega \times B)=\Omega \times \tau(B)$ for all $B \in T$. In fact we shall prove that if $\left[\psi_{1}(\Omega \times D)\right]_{\omega} \subseteq \tau\left(\left[\psi_{1}(\Omega \times D)\right]_{\omega}\right)$ for all $D \in T$ and all $\omega \in \Omega$, then (i) holds true. So let $B \in T$. We have $\Omega \times \tau(B) \subseteq \psi_{1}(\Omega \times B)$ and so

$$
\tau(B) \subseteq\left[\psi_{1}(\Omega \times B)\right]_{\omega} \subseteq \tau\left(\left[\psi_{1}(\Omega \times B)\right]_{\omega}\right)
$$

for all $\omega \in \Omega$. Now, since $\psi_{1}(\Omega \times B) \cap \psi_{1}\left(\Omega \times B^{\mathrm{c}}\right)=\emptyset$, we have

$$
\left[\psi_{1}(\Omega \times B)\right]_{\omega} \cap\left[\psi_{1}\left(\Omega \times B^{\mathrm{c}}\right)\right]_{\omega}=\emptyset .
$$

Consequently, $\tau\left(B^{\mathrm{c}}\right) \cap\left[\psi_{1}(\Omega \times B)\right]_{\omega}=\emptyset$, and so $\tau\left(\left[\psi_{1}(\Omega \times B)\right]_{\omega}\right) \subseteq \tau\left(\left[\tau\left(B^{\mathrm{c}}\right)\right]^{\mathrm{c}}\right)$. This yields

$$
\tau(B) \subseteq\left[\psi_{1}(\Omega \times B)\right]_{\omega} \subseteq \tau\left(\left[\psi_{1}(\Omega \times B)\right]_{\omega}\right) \subseteq \tau\left(\left[\tau\left(B^{\mathrm{c}}\right)\right]^{\mathrm{c}}\right)=\tau(B) .
$$

This clearly proves the validity of (i) since by the multiplicativity of $\psi_{1}$, we have

$$
\psi_{1}(A \times B)=\psi_{1}(A \times \Theta) \cap \psi_{1}(\Omega \times B)=\widetilde{\delta}(A) \times \tau(B) .
$$

REMARK 2.10. It is natural to ask whether the conditions (ii) and (iii) of Theorem 2.9 are satisfied for $\Theta$-sections. One can also ask whether the inclusion in the condition (i) may be replaced by equality. Unfortunately, the answers to these questions are negative (see Section 3).

In general, even if $\delta$ and $\tau$ are liftings, the properties (ii)-(iv) of Theorem 2.9 are not sufficient to guarantee the product property.

EXAMPLE 2.11. Let $\Omega=\{0,1\}, \Theta=[0,1), \nu$ be the Lebesgue measure on the Lebesgue measurable subsets $\Sigma$ of $[0,1)$ and $\tau$ be a lifting on the unit interval. Let $\mu$ be the purely atomic measure defined by $\mu(\{0\})=1$ and $\mu(\{1\})=0$. We define $\psi$ on the product measure space by setting for each measurable $E$,

$$
[\psi(E)]_{\{0\}}=\tau\left(E_{\{0\}}\right) \text { and }[\psi(E)]_{\{1\}}=\tau\left(E_{\{0\}}+1 / 2\right) .
$$

It is obvious that $\psi \in \Lambda(\mu \widehat{\otimes} \nu)$ and satisfies the conditions (ii)-(iv) of Theorem 2.9 but it is not a product lifting.

Definition 2.12. Let $\tau \in \vartheta(\nu)$ be an arbitrary density on $(\Theta, T, \nu)$. It is well known by [15] that

$$
\mathcal{F}(\theta):=\{B \in T: \theta \in \tau(B)\}
$$


is a filter basis in $T$ for each $\theta \in \Theta$. Hence we can choose an ultrafilter $\mathcal{U}(\theta)$ in $T$ finer than $\mathcal{F}(\theta)$. Let

$$
\sigma(B):=\{\theta \in \Theta: B \in \mathcal{U}(\theta)\} \quad \text { for all } B \in T,
$$

It has been proven in [15] that if $\nu$ is complete, then $\sigma \in \Lambda(\nu)$ and

$$
\tau(B) \subseteq \sigma(B) \subseteq\left[\tau\left(B^{\mathrm{c}}\right)\right]^{\mathrm{c}} \quad \text { for all } B \in T .
$$

If the density $\tau$ above is admissible, then $\sigma$ is called the lifting admissibly generated by the density $\tau$. The family of all admissibly generated liftings on $(\Theta, T, \nu)$ is denoted by $A G \Lambda(\nu)$. We shall prove in Theorem 4.3 that no lifting on a non-atomic measure space is an admissible density.

Since each element of $A G \Lambda(\nu)$ is consistent (see [8], Corollary 3.8), it follows that $A G \Lambda(\nu)$ is a subclass of all consistent liftings for $\nu$. This raises the question whether $A G \Lambda(\nu)$ is a proper subclass of the class of all consistent liftings.

Theorem 2.13. Let $(\Omega, \Sigma, \mu)$ and $(\Theta, T, \nu)$ be complete probability spaces. For each $\sigma \in A G \Lambda(\nu)$ and each $\varrho \in \Lambda(\mu)$, there exists $\pi_{1} \in \Lambda(\mu \widehat{\otimes} \nu)$ such that:

(i) $\pi_{1}(A \times B)=\varrho(A) \times \sigma(B)$ for all $A \in \Sigma$ and $B \in T$;

(ii) $\left[\pi_{1}(E)\right]_{\omega}=\sigma\left(\left[\pi_{1}(E)\right]_{\omega}\right)$ for all $\omega \in \Omega$ and $E \in \Sigma \widehat{\otimes} T$.

Equivalently, for each $f \in \mathcal{L}^{\infty}(\mu \widehat{\otimes} \nu)$ and each $\omega \in \Omega$,

$$
\left[\pi_{1}(f)\right]_{\omega}=\sigma\left(\left[\pi_{1}(f)\right]_{\omega}\right)
$$

and

$$
\pi_{1}(g \otimes h)=\varrho(g) \otimes \sigma(h) \quad \text { for all } g \in \mathcal{L}^{\infty}(\mu) \text { and } h \in \mathcal{L}^{\infty}(\nu) .
$$

Proof. If $\tau \in A \vartheta(\nu)$ is a density generating $\sigma$ and $\varrho \in \Lambda(\mu)$ then, according to Theorem 2.9, we can find $\psi_{1} \in \vartheta(\mu \widehat{\otimes} \nu)$ such that for all $E \in \Sigma \widehat{\otimes} T$,

$$
\begin{gathered}
{\left[\psi_{1}(E)\right]_{\omega}=\tau\left(\left[\psi_{1}(E)\right]_{\omega}\right) \quad \text { for all } \omega \in \Omega,} \\
\nu\left(\left[\psi_{1}(E)\right]_{\omega} \cup\left[\psi_{1}\left(E^{\mathrm{c}}\right)\right]_{\omega}\right)=1 \quad \text { for all } \omega \in \Omega,
\end{gathered}
$$

and

$$
\varrho(A) \times \tau(B) \subseteq \psi_{1}(A \times B) \quad \text { for each } A \in \Sigma \text { and } B \in T .
$$

We now define $\pi_{1} \in \vartheta(\mu \widehat{\otimes} \nu)$ by setting for each $E \in \Sigma \widehat{\otimes} T$ and each $\omega \in \Omega$,

$$
\left[\pi_{1}(E)\right]_{\omega}=\sigma\left(\left[\psi_{1}(E)\right]_{\omega}\right) \text {. }
$$

To prove that $\pi_{1}$ is a lifting, notice that by $(6)$ we get $\left[\pi_{1}\left(E^{\mathrm{c}}\right)\right]_{\omega}=\left(\left[\pi_{1}(E)\right]_{\omega}\right)^{\mathrm{c}}$ $\nu$-almost everywhere for each $\omega$. Hence

$$
\sigma\left(\left[\pi_{1}\left(E^{\mathrm{c}}\right)\right]_{\omega}\right)=\sigma\left[\left(\left[\pi_{1}(E)\right]_{\omega}\right)^{\mathrm{c}}\right] .
$$


Taking into account (8), (9) and the lifting properties of $\sigma$ we see that

$$
\left[\pi_{1}\left(E^{\mathrm{c}}\right)\right]_{\omega}=\left(\left[\pi_{1}(E)\right]^{\mathrm{c}}\right)_{\omega} .
$$

This implies $\pi_{1}\left(E^{\mathrm{c}}\right)=\left[\pi_{1}(E)\right]^{\mathrm{c}}$ and so $\pi_{1} \in \Lambda(\mu \widehat{\otimes} \nu)$.

In order to show (i), let $A \times B \in \Sigma \otimes T$. Then, according to (7) and (8),

$$
\begin{aligned}
{\left[\pi_{1}(A \times B)\right]_{\omega} } & =\sigma\left(\left[\psi_{1}(A \times B)\right]_{\omega}\right) \supseteq \sigma\left([\varrho(A) \times \tau(B)]_{\omega}\right) \\
& = \begin{cases}\sigma(B) & \text { if } \omega \in \varrho(A), \\
\emptyset & \text { if } \omega \notin \varrho(A) .\end{cases}
\end{aligned}
$$

Applying the same considerations to the sets $A \times B^{\mathrm{c}}, A^{\mathrm{c}} \times B, A^{\mathrm{c}} \times B^{\mathrm{c}}$ we get

$$
\pi_{1}(A \times B)=\varrho(A) \times \sigma(B) .
$$

In general the liftings $\pi_{1}$ and $\pi_{2}$ coming from Theorems 2.13 and $2.13^{\perp}$, respectively, will be different (cf. Theorem 3.5). If $(\Omega, \Sigma, \mu)=(\Theta, T, \nu)$ and $\varrho=\sigma$, then it is easily seen that the equality $\pi_{1}=\pi_{2}$ would yield the existence of a symmetric lifting, that is, a lifting $\pi \in \Lambda(\mu \widehat{\otimes} \nu)$ with the property

$$
\pi(E)=\left[\pi\left(E^{\mathrm{s}}\right)\right]^{\mathrm{s}},
$$

where $E^{\mathrm{s}}=\{(\omega, \theta):(\theta, \omega) \in E\}$. In the case of non-atomic $\mu$ this is however impossible.

3. Existence of liftings and densities with lifting invariant or density invariant sections. There is now a question whether there exist $\varrho \in \Lambda(\mu), \sigma \in \Lambda(\nu)$ and $\pi \in \Lambda(\mu \widehat{\otimes} \nu)$ such that for each $E \in \Sigma \widehat{\otimes} T$ and each $(\omega, \theta) \in \Omega \times \Theta$,

$$
\varrho\left([\pi(E)]^{\theta}\right)=[\pi(E)]^{\theta} \quad \text { and } \quad \sigma\left([\pi(E)]_{\omega}\right)=[\pi(E)]_{\omega} .
$$

An easy calculation shows that (10) yields $\pi \in \varrho \otimes \sigma$.

It is our aim to prove that the above question has in general a negative answer. In particular, it is so in the case of non-atomic measures $\mu$ and $\nu$.

We start however with some positive results.

Theorem 3.1. For each $\delta \in A \vartheta(\mu)$ and each $\tau \in A \vartheta(\nu)$ there exists $\varphi \in \vartheta(\mu \otimes \nu)$ such that $\varphi \in \delta \otimes \tau$ and for each $E \in \Sigma \otimes T$ there exist $N_{E} \in \Sigma_{0}$ and $M_{E} \in T_{0}$ such that

$$
[\varphi(E)]_{\omega} \subseteq \tau\left([\varphi(E)]_{\omega}\right) \quad \text { for all } \omega \in \Omega \backslash N_{E},
$$

and

$$
[\varphi(E)]^{\theta} \subseteq \delta\left([\varphi(E)]^{\theta}\right) \quad \text { for all } \theta \in \Theta \backslash M_{E} .
$$

If $(\Omega, \Sigma, \mu)=(\Theta, T, \nu)$ and $\delta=\tau$, then $\varphi \in \delta \otimes \delta$ can be chosen to be symmetric. 
Pro of. According to Theorem 2.5 we get $\varphi_{1} \in \delta \otimes \tau$ such that for every $E \in \Sigma \otimes T$ there exists $N_{E} \in \Sigma_{0}$ with

$$
\left[\varphi_{1}(E)\right]_{\omega}=\tau\left(\left[\varphi_{1}(E)\right]_{\omega}\right) \quad \text { for all } \omega \in \Omega \backslash N_{E} .
$$

Applying Theorem $2.5^{\perp}$ we get $\varphi_{2} \in \delta \otimes \tau$ such that for every $E \in \Sigma \otimes T$ there exists $M_{E} \in T$ with

$$
\left[\varphi_{2}(E)\right]^{\theta}=\delta\left(\left[\varphi_{2}(E)\right]^{\theta}\right) \quad \text { for all } \theta \in \Theta \backslash M_{E} .
$$

Since $\varphi_{1}$ and $\varphi_{2}$ are densities for the same measure, for each $E \in \Sigma \otimes T$ there exists a set $\widetilde{N}_{E} \in \Sigma_{0}$ such that

$$
\mu\left(\left[\varphi_{1}(E)\right]_{\omega} \triangle\left[\varphi_{2}(E)\right]_{\omega}\right)=0
$$

for all $\omega \in \Omega \backslash \widetilde{N}_{E}$. Now define $\varphi \in \vartheta(\mu \otimes \nu)$ by setting

$$
\varphi(E):=\varphi_{1}(E) \cap \varphi_{2}(E) .
$$

It then follows from (11) and (12) that for each $\omega \notin \widetilde{N}_{E} \cup N_{E}$,

$$
[\varphi(E)]_{\omega} \subseteq\left[\varphi_{1}(E)\right]_{\omega}=\tau\left(\left[\varphi_{1}(E)\right]_{\omega}\right)=\tau\left([\varphi(E)]_{\omega}\right) .
$$

Similarly one can get the inclusion $[\varphi(E)]^{\theta} \subseteq \delta\left([\varphi(E)]^{\theta}\right)$ for almost all $\theta$.

In the case of $\delta=\tau$ we take, in the above proof, $\widetilde{\varphi}_{1}(E)=\left[\varphi_{1}\left(E^{\mathrm{s}}\right)\right]^{\mathrm{s}}$ for all $E \in \Sigma \otimes \Sigma$ instead of $\varphi_{2}$ and set $\varphi(E):=\varphi_{1}(E) \cap \widetilde{\varphi}_{1}(E)$.

Theorem 3.2. If $(\Omega, \Sigma, \mu)$ and $(\Theta, T, \nu)$ are complete, then for each $\delta \in A \vartheta(\mu)$ and each $\tau \in A \vartheta(\nu)$ there exists $\widetilde{\varphi} \in \vartheta(\mu \widehat{\otimes} \nu)$ such that $\widetilde{\varphi} \in \delta \otimes \tau$ and for each $E \in \Sigma \widehat{\otimes} T$ and each $(\omega, \theta) \in \Omega \times \Theta$ we have

$$
[\widetilde{\varphi}(E)]_{\omega} \subseteq \tau\left([\widetilde{\varphi}(E)]_{\omega}\right) \text { and }[\widetilde{\varphi}(E)]^{\theta} \subseteq \delta\left([\widetilde{\varphi}(E)]^{\theta}\right) .
$$

If $(\Omega, \Sigma, \mu)=(\Theta, T, \nu)$ and $\delta=\tau$, then $\widetilde{\varphi} \in \delta \widehat{\otimes} \delta$ can be chosen to be symmetric.

Proof. Let $\bar{\varphi}_{1}$ and $\bar{\varphi}_{2}$ satisfy the conditions of Corollary 2.7 and $2.7^{\perp}$. Define $\check{\varphi}_{1}, \check{\varphi}_{2} \in \vartheta(\mu \widehat{\otimes} \nu)$ by setting for each $\omega \in \Omega, \theta \in \Theta$ and $E \in \Sigma \widehat{\otimes} T$,

$$
\left[\check{\varphi}_{1}(E)\right]_{\omega}:=\tau\left(\left[\bar{\varphi}_{2}(E)\right]_{\omega}\right) \text { and }\left[\check{\varphi}_{2}(E)\right]^{\theta}:=\delta\left(\left[\bar{\varphi}_{1}(E)\right]^{\theta}\right) \text {. }
$$

In a standard way one can check the correctness of the definitions. It is a consequence of the Fubini Theorem that there exists $M_{E} \in \Sigma_{0}$ such that for each $\omega \notin M_{E}$ the sets $\left[\check{\varphi}_{1}(E)\right]_{\omega}$ and $\left[\bar{\varphi}_{1}(E)\right]_{\omega}$ are measure equivalent. Since they are density invariant, they are equal. Consequently, for each $\theta \in \Theta$ we get

$$
\delta\left(\left[\check{\varphi}_{1}(E)\right]^{\theta}\right)=\delta\left(\left[\bar{\varphi}_{1}(E)\right]^{\theta}\right)=\left[\check{\varphi}_{2}(E)\right]^{\theta} .
$$

In a similar way, for each $\omega$ we can get

$$
\tau\left(\left[\check{\varphi}_{2}(E)\right]_{\omega}\right)=\tau\left(\left[\bar{\varphi}_{2}(E)\right]_{\omega}\right)=\left[\check{\varphi}_{1}(E)\right]_{\omega} .
$$


Now define $\widetilde{\varphi} \in \vartheta(\mu \widehat{\otimes} \nu)$ by setting

$$
\widetilde{\varphi}(E):=\check{\varphi}_{1}(E) \cap \check{\varphi}_{2}(E) .
$$

For each $E \in \Sigma \widehat{\otimes} T, \theta \in \Theta$ and $\omega \in \Omega$ we have

$$
\tau\left([\widetilde{\varphi}(E)]_{\omega}\right)=\tau\left(\left[\check{\varphi}_{1}(E)\right]_{\omega}\right) \cap \tau\left(\left[\check{\varphi}_{2}(E)\right]_{\omega}\right)=\left[\check{\varphi}_{1}(E)\right]_{\omega}
$$

and

$$
\delta\left([\widetilde{\varphi}(E)]^{\theta}\right)=\left[\check{\varphi}_{2}(E)\right]^{\theta} .
$$

It follows that

$$
[\widetilde{\varphi}(E)]_{\omega} \subseteq \tau\left([\widetilde{\varphi}(E)]_{\omega}\right) \text { and }[\widetilde{\varphi}(E)]^{\theta} \subseteq \delta\left([\widetilde{\varphi}(E)]^{\theta}\right) .
$$

REMARK 3.3. We can consider the collection of all (symmetric) densities satisfying the conclusion of Theorem 3.2 and partially ordered by inclusion. With the help of the Zorn-Kuratowski Lemma one can prove the existence of a maximal element $\psi$ such that for each $E \in \Sigma \widehat{\otimes} T$ and for all $\omega \in \Omega$ and $\theta \in \Theta$,

$$
[\psi(E)]_{\omega} \subseteq \tau\left([\psi(E)]_{\omega}\right) \text { and }[\psi(E)]^{\theta} \subseteq \delta\left([\psi(E)]^{\theta}\right) .
$$

Notice that for each $E \in \Sigma \widehat{\otimes} T$ and for all $\omega \in \Omega$ we have

$$
\text { either } \quad[\psi(E)]_{\omega}=\emptyset \quad \text { or } \quad \nu\left([\psi(E)]_{\omega}\right)>0 .
$$

A similar assertion holds true also for all $\theta \in \Theta$.

We will prove however that the inclusions in (15) cannot be replaced by equalities.

In what follows we use the notation $\varphi=\delta \otimes_{\mathrm{F}} \tau$ if, for each $E \in \Sigma \widehat{\otimes} T$, $\omega \in \Omega$ and $\theta \in \Theta$ the equalities

$$
[\varphi(E)]_{\omega}=\tau\left([\varphi(E)]_{\omega}\right) \text { and }[\varphi(E)]^{\theta}=\delta\left([\varphi(E)]^{\theta}\right)
$$

hold true, where $\delta \in \vartheta(\mu), \tau \in \vartheta(\nu)$ and $\varphi \in \vartheta(\mu \widehat{\otimes} \nu)$. The letter $\mathrm{F}$ in $\otimes_{\mathrm{F}}$ stands for Fubini, since it would be quite natural to call such a density $\varphi$ a Fubini density.

Now we are going to answer the question posed at the beginning of the section. We start with an easy lemma.

Lemma 3.4. Let $(\Omega, \Sigma, \mu)$ be an arbitrary non-atomic probability space and let $\delta \in \vartheta(\mu)$. Then $\inf \{\mu(A): \omega \in \delta(A)\}=0$ for $\mu$-almost all $\omega \in \Omega$.

TheOREM 3.5. If $(\Omega, \Sigma, \mu)$ and $(\Theta, T, \nu)$ are not purely atomic probability spaces then there do not exist densities $\delta \in \vartheta(\mu), \tau \in \vartheta(\nu)$ and $\varphi \in \vartheta(\mu \widehat{\otimes} \nu)$ such that $\varphi=\delta \otimes_{\mathrm{F}} \tau$.

Proof. Assume that $\mu$ and $\nu$ are non-atomic and we have $\delta \in \vartheta(\mu)$, $\tau \in \vartheta(\nu)$ and $\varphi \in \vartheta(\mu \widehat{\otimes} \nu)$ such that $\varphi=\delta \otimes_{\mathrm{F}} \tau$. Fix $\omega \in \Omega$ and $\theta \in \Theta$ 
such that

$$
\inf \{\mu(A): \omega \in \delta(A)\}=0 \quad \text { and } \inf \{\nu(B): \theta \in \tau(B)\}=0 .
$$

Then take two decreasing sequences $\left\langle\widetilde{A}_{n, 1}\right\rangle$ and $\left\langle\widetilde{B}_{n, 1}\right\rangle$ in $\Sigma$ and $T$, respectively, such that

$$
\begin{aligned}
& \mu\left(\widetilde{A}_{n, 1}\right)>0 \quad \text { and } \quad \nu\left(\widetilde{B}_{n, 1}\right)>0 \quad \text { for each } n \in \mathbb{N} ; \\
& \omega \in \widetilde{A}_{n, 1}=\delta\left(\widetilde{A}_{n, 1}\right) \quad \text { and } \quad \theta \in \widetilde{B}_{n, 1}=\tau\left(\widetilde{B}_{n, 1}\right) \quad \text { for each } n \in \mathbb{N} \text {; }
\end{aligned}
$$

and

$$
\lim _{n} \mu\left(\widetilde{A}_{n, 1}\right)=0 \quad \text { and } \quad \lim _{n} \nu\left(\widetilde{B}_{n, 1}\right)=0
$$

If $\mathcal{F}(\omega)$ and $\mathcal{F}(\theta)$ are the filters generated by $(\delta, \omega)$ and $(\tau, \theta)$ respectively, then $\widetilde{A}_{n, 1} \in \mathcal{F}(\omega)$ and $\widetilde{B}_{n, 1} \in \mathcal{F}(\theta)$ for all $n \in \mathbb{N}$.

Now set

$$
\widetilde{A}_{1,0}:=\Omega \backslash \widetilde{A}_{1,1} \quad \text { and } \quad \widetilde{B}_{1,0}:=\Theta \backslash \widetilde{B}_{1,1} ;
$$

and then for each $n \in \mathbb{N}$,

$$
\widetilde{A}_{n+1,0}:=\widetilde{A}_{n, 1} \backslash \widetilde{A}_{n+1,1} \quad \text { and } \quad \widetilde{B}_{n+1,0}:=\widetilde{B}_{n, 1} \backslash \widetilde{B}_{n+1,1} .
$$

Define new sequences of sets by setting

$$
\begin{aligned}
A_{1,0} & :=\widetilde{A}_{1,0} \cup \bigcap_{n=1}^{\infty} \widetilde{A}_{n, 1} \quad \text { and } \quad B_{1,0}:=\widetilde{B}_{1,0} \cup \bigcap_{n=1}^{\infty} \widetilde{B}_{n, 1} ; \\
A_{n 1} & :=\widetilde{A}_{n, 1} \backslash \bigcap_{k=1}^{\infty} \widetilde{A}_{k, 1} \quad \text { and } \quad B_{n 1}:=\widetilde{B}_{n, 1} \backslash \bigcap_{k=1}^{\infty} \widetilde{B}_{k, 1}
\end{aligned}
$$

and

$$
A_{n+1,0}:=A_{n 1} \backslash A_{n+1,1} \quad \text { and } \quad B_{n+1,0}:=B_{n 1} \backslash B_{n+1,1}
$$

whenever $n \geq 1$. Since the sets $\bigcap_{n=1}^{\infty} \widetilde{A}_{n, 1}$ and $\bigcap_{n=1}^{\infty} \widetilde{B}_{n, 1}$ are of measure zero and the filters $\mathcal{F}(\omega)$ and $\mathcal{F}(\theta)$ are measure stable we have $A_{n 1} \in \mathcal{F}(\omega)$ and $B_{n 1} \in \mathcal{F}(\theta)$. Thus, we have got the sequences $\left\langle A_{n i}\right\rangle$ and $\left\langle B_{n i}\right\rangle$ with $i \in\{0,1\}$ satisfying for every $n \in \mathbb{N}$ the following conditions:

$$
\begin{gathered}
A_{n 0} \cap A_{n 1}=\emptyset \quad \text { and } A_{n+1,0} \cup A_{n+1,1}=A_{n 1} ; \\
B_{n 0} \cap B_{n 1}=\emptyset \text { and } B_{n+1,0} \cup B_{n+1,1}=B_{n 1} ; \\
\bigcup_{n=1}^{\infty} A_{n 0}=\Omega \quad \text { and } \bigcup_{n=1}^{\infty} B_{n 0}=\Theta ; \\
\bigcap_{n=1}^{\infty} A_{n 1}=\emptyset \quad \text { and } \bigcap_{n=1}^{\infty} B_{n 1}=\emptyset ; \\
A_{n 1} \in \mathcal{F}(\omega) \text { and } B_{n 1} \in \mathcal{F}(\theta) .
\end{gathered}
$$


We define two new sets in the product space by setting

$$
U:=\bigcup_{n=1}^{\infty} A_{n 0} \times B_{n 1} \text { and } L:=\bigcup_{n=1}^{\infty} A_{n 1} \times B_{n 0} .
$$

We have $U \cap L=\emptyset$.

Now if $\bar{\omega} \in \Omega$ then there is $n_{\bar{\omega}} \in \mathbb{N}$ such that $\bar{\omega} \in A_{n_{\bar{\omega}} 0}$ and so

$$
U_{\bar{\omega}}=\left(\bigcup_{n=1}^{\infty} A_{n 0} \times B_{n 1}\right)_{\bar{\omega}}=\left(A_{n_{\bar{\omega}} 0} \times B_{n_{\bar{\omega}} 1}\right)_{\bar{\omega}}=B_{n_{\varpi} 1} \in \mathcal{F}(\theta) .
$$

Hence $U_{\bar{\omega}} \in \mathcal{F}(\theta)$. Similarly $L^{\bar{\theta}} \in \mathcal{F}(\omega)$. Applying now the equality $\varphi=\delta \otimes_{\mathrm{F}} \tau$ one can easily see that $(\omega, \theta) \in \varphi(U) \cap \varphi(L)$. This is however impossible since $U \cap L=\emptyset$ and so $\varphi(U) \cap \varphi(L)=\emptyset$.

This completes the whole proof.

In the case of atomic measure spaces liftings with lifting invariant sections may exist.

EXAmple 3.6. Consider a complete $(\Theta, T, \nu)$ and $(\Omega, \Sigma, \mu)$ such that $\Omega=\bigcup_{n}\left\{\omega_{n}\right\}$ and each point $\omega_{n}$ is of positive measure. Given $\sigma \in \Lambda(\nu)$ define a lifting $\pi \in \Lambda(\mu \widehat{\otimes} \nu)$ by

$$
\pi(E):=\bigcup_{n}\left\{\omega_{n}\right\} \times \sigma\left(E_{\omega_{n}}\right) .
$$

Then $\pi=\varrho \otimes_{\mathrm{F}} \sigma$, where $\varrho$ is the unique lifting on the family of all subsets of $\Omega$.

4. Existence of liftings with measurable sections. As we have proven in previous sections, when admissible densities are under consideration, there always exist product densities with nice measurability properties. In Theorem 2.9 we have proven the existence of a product density with density invariant $\Omega$-sections and measurable $\Theta$-sections. In Theorem 3.2 the existence of a product density with measurable sections satisfying an inclusion has been proven. There is now a question whether similar results can be achieved for liftings; in particular, whether the lifting $\pi_{1}$ in Theorem 2.13 can have all $\Theta$-sections measurable. More generally: do there exist $\varrho \in \Lambda(\mu), \sigma \in \Lambda(\nu)$ and $\pi \in \Lambda(\mu \widehat{\otimes} \nu)$ such that $\pi \in \varrho \otimes \sigma$ and for each $E \in \Sigma \widehat{\otimes} T$ and $(\omega, \theta) \in \Omega \times \Theta$,

$$
\sigma\left([\pi(E)]_{\omega}\right)=[\pi(E)]_{\omega} \text { and }[\pi(E)]^{\theta} \text { is measurable. }
$$

Assuming the existence of real-valued measurable cardinals and taking $\mu$ to be a universal measure on $\Omega$ of measurable cardinality, we get an example of $\pi_{1}$ with all sections measurable. In general however $\pi_{1}$ with measurable $\Theta$-sections and lifting invariant $\Omega$-sections does not exist. The full description of liftings satisfying (16) remains an open problem. 
We start with the following result of Blass [2]:

Proposition 4.1. Let $X$ be the countable product of the two-point space $\{0,1\}$ equipped with the ordinary product Haar measure $\lambda$ and let $\mathcal{L}$ be the $\sigma$-algebra of $\lambda$-measurable sets. Let $(\Theta, T, \nu)$ be a complete non-atomic probability space. If $\mathcal{V}$ is a $\nu$-stable ultrafilter in $T$ then there exists a set $E \in \mathcal{L} \otimes T$ such that

$$
\left\{x \in X: E_{x} \in \mathcal{V}\right\} \notin \mathcal{L} .
$$

Proof. We first prove that if

$$
\left\{x \in X: E_{x} \in \mathcal{V}\right\} \in \mathcal{L}
$$

for every $E \in \mathcal{L} \otimes T$, then for each $B \in \mathcal{V}$ such that $B=\bigcup_{n=1}^{\infty} B_{n}$ and all $B_{n}$ are measurable and pairwise disjoint, there is $n \in \mathbb{N}$ with $B_{n} \in \mathcal{V}$. So consider $B=\bigcup_{n=1}^{\infty} B_{n} \in \mathcal{V}$ with all $B_{n}$ 's being measurable and non-empty. Then let

$$
E:=\bigcup_{n}\left(\left\{\left\langle x_{m}\right\rangle \in X: x_{n}=1\right\} \times B_{n}\right) .
$$

Since $E \in \mathcal{L} \otimes T$ we may apply (18). For each $x \in X$ let $\widehat{x} \subset \mathbb{N}$ be such that $x=\chi_{\widehat{x}}$. We can now write for each $x \in X$,

$$
E_{x}=\bigcup_{\left\{n: x_{n}=1\right\}} B_{n}=\bigcup_{n \in \widehat{x}} B_{n} .
$$

Since $\mathcal{V}$ is an ultrafilter and the sets $B_{n}$ are pairwise disjoint, and $B \in \mathcal{V}$, the collection

$$
\mathcal{W}:=\left\{Z \subseteq \mathbb{N}: \bigcup_{n \in Z} B_{n} \in \mathcal{V}\right\}
$$

is an ultrafilter on $\mathbb{N}$. Applying (18) and (19) we get

$$
\{x \in X: \widehat{x} \in \mathcal{W}\}=\left\{x \in X: E_{x} \in \mathcal{V}\right\} \in \mathcal{L} .
$$

But according to a theorem of Sierpiński [10], if $\mathcal{W}$ is a free ultrafilter on $\mathbb{N}$ then the set $\{x \in X: \widehat{x} \in \mathcal{W}\}$ is non-measurable with respect to $\lambda$. Thus $\mathcal{W}$ must be fixed, which simply means that there exists $n$ with $B_{n} \in \mathcal{V}$.

Now we can prove the main assertion. To do it divide $\Theta$ into two disjoint sets of equal measure. Then divide each piece again into two disjoint sets of equal measure and so on. It follows from the basic properties of ultrafilters that we thus obtain a decreasing sequence $\left\langle C_{n}\right\rangle$ of sets such that $C_{n} \in \mathcal{V}$ for each $n$. Moreover

$$
C_{1}=\bigcup_{n=1}^{\infty}\left(C_{n} \backslash C_{n+1}\right) \cup \bigcap_{n=1}^{\infty} C_{n}
$$

and $\nu\left(\bigcap_{n=1}^{\infty} C_{n}\right)=0$. It follows from the first part of the proof that 
$\left\{x \in X: E_{x} \in \mathcal{V}\right\} \notin \mathcal{L}$ for some $E \in \mathcal{L} \widehat{\otimes} T$ (otherwise $\bigcap_{n=1}^{\infty} C_{n} \in \mathcal{V}$, which would contradict the $\nu$-stability of $\mathcal{V}$ ).

From the above proposition one can easily deduce a more general result:

Proposition 4.2. Let $(\Omega, \Sigma, \mu)$ be a complete non-atomic and perfect probability space and let $(\Theta, T, \nu)$ be a complete non-atomic probability space. If $\mathcal{V}$ is a $\nu$-stable ultrafilter in $T$ then there exists a set $E \in \Sigma \widehat{\otimes} T$ such that

$$
\left\{\omega \in \Omega: E_{\omega} \in \mathcal{V}\right\} \notin \Sigma .
$$

Proof. There exists a measurable surjection $h: \Omega \rightarrow X$ such that $\mu\left(h^{-1}(B)\right)=\lambda(B)$ for each $B \in \mathcal{L}$ (cf. [7]). By Proposition 4.1, there is $F \in \mathcal{L} \otimes T$ such that $\left\{x \in X: F_{x} \in \mathcal{V}\right\} \notin \mathcal{L}$. Let $\widetilde{h}: \Omega \times \Theta \rightarrow X \times \Theta$ be given by $\widetilde{h}(\omega, \theta)=(h(\omega), \theta)$. It easily follows that $\widetilde{h}^{-1}(\mathcal{L} \otimes T) \subseteq \Sigma \widehat{\otimes} T$. Set $E:=\widetilde{h}^{-1}(F)$. We have

$$
\begin{aligned}
\left\{\omega \in \Omega: E_{\omega} \in \mathcal{V}\right\} & =\left\{\omega \in \Omega:\left[\widetilde{h}^{-1}(F)\right]_{\omega} \in \mathcal{V}\right\}=\left\{\omega \in \Omega: F_{h(\omega)} \in \mathcal{V}\right\} \\
& =h^{-1}\left\{x \in X: F_{x} \in \mathcal{V}\right\} .
\end{aligned}
$$

It now follows from the perfectness of $\mu$ and the non-measurability of the set $\left\{x \in X: F_{x} \in \mathcal{V}\right\}$ that $\left\{\omega \in \Omega: E_{\omega} \in \mathcal{V}\right\} \notin \Sigma$.

Applying Proposition 4.2 we obtain the main non-existence result of this paper.

THEOREM 4.3. Let $(\Omega, \Sigma, \mu)$ be a complete non-atomic and perfect probability space and let $(\Theta, T, \nu)$ be a complete non-atomic probability space. There exist no liftings $\sigma \in \Lambda(\nu)$ and $\pi \in \Lambda(\mu \widehat{\otimes} \nu)$ satisfying the following two conditions:

(j) there exists $\bar{\theta} \in \Theta$ such that for each $E \in \Sigma \widehat{\otimes} T$,

$$
[\pi(E)]^{\bar{\theta}} \in \Sigma .
$$

(jj) for each $E \in \Sigma \widehat{\otimes} T$ there exists a set $N_{E} \in \Sigma_{0}$ such that

$$
[\pi(E)]_{\omega}=\sigma\left([\pi(E)]_{\omega}\right) \quad \text { for each } \omega \notin N_{E} .
$$

In particular, no $\sigma \in \Lambda(\nu)$ is an admissible density.

Proof. Suppose that liftings satisfying all the above assumptions exist and let $\langle\mathcal{V}(\theta)\rangle_{\theta \in \Theta}$ be the family of ultrafilters generating $\sigma$. If $E \in \Sigma \widehat{\otimes} T$, then by the Fubini Theorem there exists a set $K_{E} \in \Sigma_{0}$ such that

$$
[\pi(E)]_{\omega}=E_{\omega} \quad \text { a.e. }(\nu) \text {, for each } \omega \notin K_{E} .
$$

Set $L_{E}=K_{E} \cup N_{E}$. Then for each $\theta \in \Theta$,

$$
\left\{\omega \in \Omega: E_{\omega} \in \mathcal{V}(\theta)\right\} \cap\left(\Omega \backslash L_{E}\right)=\left(\Omega \backslash L_{E}\right) \cap[\pi(E)]^{\theta} .
$$


Since $(\Omega, \Sigma, \mu)$ is complete and $[\pi(E)]^{\bar{\theta}} \in \Sigma$, we have

$$
\left\{\omega \in \Omega: E_{\omega} \in \mathcal{V}(\bar{\theta})\right\} \in \Sigma
$$

for every $E \in \Sigma \widehat{\otimes} T$. This however contradicts Proposition 4.2 since each $\mathcal{V}(\theta)$ is measure stable (because $\sigma$ is a lifting).

Corollary 4.4. Let $(\Omega, \Sigma, \mu)$ and $(\Theta, T, \nu)$ be as in Proposition 4.2 and let $\varrho, \sigma$ and $\pi_{1}$ be liftings satisfying the conclusion of Theorem 2.13. Then for each $\theta \in \Theta$ there exists $E \in \Sigma \widehat{\otimes} T$ such that $\left[\pi_{1}(E)\right]^{\theta}$ is non-measurable.

It follows from the above corollary that Theorem 2.13 is the best possible result, at least when $\nu$ is perfect.

Corollary 4.5. Let $(\Omega, \Sigma, \mu)$ be a complete non-atomic probability space and let $(\Theta, T, \nu)$ be a complete non-atomic and perfect probability space. Furthermore, let $\varrho \in \Lambda(\mu), \sigma \in A G \Lambda(\nu)$ and $\pi_{1} \in \Lambda(\mu \widehat{\otimes} \nu)$ be liftings satisfying the conclusion of Theorem 2.13. Then there exists $E \in \Sigma \widehat{\otimes} T$ such that

$$
\nu^{*}\left\{\theta \in \Theta:\left[\pi_{1}(E)\right]^{\theta} \neq \varrho\left(\left[\pi_{1}(E)\right]^{\theta}\right)\right\}>0 .
$$

We finish with the following open problems, which seem to be interesting. In both cases we assume the non-atomicity of the measures.

Question 4.6. Do there exist $\varrho \in \Lambda(\mu), \sigma \in \Lambda(\nu)$ and $\pi \in \Lambda(\mu \widehat{\otimes} \nu)$ such that $\pi \in \varrho \otimes \sigma$ and for each $E \in \Sigma \widehat{\otimes} T$ and $(\omega, \theta) \in \Omega \times \Theta$,

$$
[\pi(E)]_{\omega} \in T \text { and }[\pi(E)]^{\theta} \in \Sigma \text { ? }
$$

Question 4.7. Do there exist $\varrho \in \Lambda(\mu), \sigma \in \Lambda(\nu)$ and $\pi \in \Lambda(\mu \widehat{\otimes} \nu)$ such that $\pi \in \varrho \otimes \sigma$ and for each $E \in \Sigma \widehat{\otimes} T$ there exist $N_{E} \in \Sigma_{0}$ and $M_{E} \in T_{0}$ with the property that whenever $\omega \notin N_{E}$ and $\theta \notin M_{E}$ then

$$
\varrho\left([\pi(E)]^{\theta}\right)=[\pi(E)]^{\theta} \text { and } \sigma\left([\pi(E)]_{\omega}\right)=[\pi(E)]_{\omega} \text { ? }
$$

5. Applications to functions of two variables and stochastic processes. Let $(\Omega, \Sigma, \mu),(\Theta, T, \nu)$ be complete probability spaces and let $X=\left\{X_{\theta}\right\}_{\theta \in \Theta}$ be an arbitrary real-valued stochastic process on $(\Omega, \Sigma, \mu)$. If $Y=\left\{Y_{\theta}\right\}_{\theta \in \Theta}$ is another stochastic process then it is called equivalent to $\left\{X_{\theta}\right\}_{\theta \in \Theta}$ if for each $\theta \in \Theta$ the equality $X_{\theta}=Y_{\theta}$ holds true a.e. $(\mu)$ (the exceptional set may depend on $\theta) .\left\{X_{\theta}\right\}_{\theta \in \Theta}$ is said to be measurable if the map $(\omega, \theta) \mapsto X_{\theta}(\omega)$ is measurable with respect to the product $\sigma$-algebra $\Sigma \widehat{\otimes} T ;\left\{X_{\theta}\right\}_{\theta \in \Theta}$ is bounded if the family $\left\{X_{\theta}: \theta \in \Theta\right\}$ is bounded in $L_{\infty}(\mu)$. There are several papers concerning the existence of measurable (or separable) processes that are equivalent to a given process (cf. [3], [14], [12]). Sometimes a measurable process equivalent to a bounded $\left\{X_{\theta}\right\}_{\theta \in \Theta}$ can be defined by setting $Y_{\theta}=\varrho\left(X_{\theta}\right)$, where $\varrho \in \Lambda(\mu)$ and the initial process $X$ or $(\Theta, T, \nu)$ satisfy some additional conditions. In particular, the 
lifting $\varrho$ in [3] is assumed to be strong and $\Omega$ is taken to be an interval. In general however, strong lifting may not exist on the topological measure space investigated. With the help of Theorem 2.13 and $2.13^{\perp}$ we get-by a different method - the following two results from [3], without any topological assumptions:

TheOREM 5.1. For each $\sigma \in A G \Lambda(\nu)$ and each bounded measurable stochastic process $\left\{X_{\theta}\right\}_{\theta \in \Theta}$ on a space $(\Omega, \Sigma, \mu)$ there is a collection $\left\{Y_{\theta}\right\}_{\theta \in \Theta}$ of measurable functions on $(\Omega, \Sigma, \mu)$ satisfying the following conditions:

(i) $Y .(\omega)=\sigma(Y .(\omega))$ for each $\omega \in \Omega$;

(ii) there is $M_{X} \in T_{0}$ such that for every $\theta \notin M_{X}$ we have $X_{\theta}=Y_{\theta}$ a.e. ( $\mu$ ) and $\left\{Y_{\theta}\right\}_{\theta \notin M_{X}}$ is a measurable stochastic process on $(\Omega, \Sigma, \mu)$;

(iii) there is $N_{X} \in \Sigma_{0}$ such that for every $\omega \notin N_{X}$ we have $X .(\omega)=Y .(\omega)$ a.e. $(\nu)$;

(iv) if $\Theta$ is a separable metric space and $\left(X_{\theta}\right)_{\theta \notin M_{X}}$ is continuous in probability, then $\left(Y_{\theta}\right)_{\theta \notin M_{X}}$ is separable; furthermore, every countable dense subset of $\Theta \backslash M_{X}$ is a separating set for $\left(Y_{\theta}\right)_{\theta \notin M_{X}}$.

Pr o o f. We take arbitrary $\sigma \in A G \Lambda(\nu), \varrho \in \Lambda(\mu)$ and the corresponding $\pi_{1} \in \Lambda(\mu \widehat{\otimes} \nu)$, existing in view of Theorem 2.13. Setting $Y:=\pi_{1}(X)$, we get a $\Sigma \widehat{\otimes} T$-measurable function with the property that for each $\omega$ the function $Y .(\omega)=\left[\pi_{1}(X)\right]_{\omega}$ fulfils the equality $Y .(\omega)=\sigma(Y .(\omega))$. This means that $\left\{Y_{\theta}\right\}_{\theta \in \Theta}$ is a stochastic process satisfying (i). The conditions (ii) and (iii) follow from the Fubini Theorem. (iv) can be proved exactly as in [3].

In the terminology of [3] the process $\left\{Y_{\theta}\right\}_{\theta \in \Theta}$ is called $\sigma$-canonical.

Theorem 5.2. For each $\varrho \in A G \Lambda(\mu)$ and each bounded measurable stochastic process $\left\{X_{\theta}\right\}_{\theta \in \Theta}$ on a space $(\Omega, \Sigma, \mu)$ there is a measurable process $\left\{Y_{\theta}\right\}_{\theta \in \Theta}$ on $(\Omega, \Sigma, \mu)$ that is equivalent to $\left\{X_{\theta}\right\}_{\theta \in \Theta}$ and satisfies the following conditions:

(i) $Y_{\theta}=\varrho\left(Y_{\theta}\right)$ for each $\theta$;

(ii) there is $N_{X} \in \Sigma_{0}$ such that for each $\omega \notin N_{X}$ we have $X .(\omega)=Y$. $(\omega)$ a.e. $(\nu)$.

Pro of. Let $\varrho \in A G \Lambda(\mu)$ and $\sigma \in \Lambda(\nu)$. In view of Theorem $2.13^{\perp}$ there exists $\pi_{2} \in \Lambda(\mu \widehat{\otimes} \nu)$ such that given a process $X=\left\{X_{\theta}\right\}_{\theta \in \Theta}$, we have

$$
\left[\pi_{2}(X)\right]^{\theta}=\varrho\left(\left[\pi_{2}(X)\right]^{\theta}\right) \quad \text { for all } \theta \in \Theta .
$$

By the Fubini Theorem there exists $M_{X} \in T_{0}$ such that

$$
X_{\theta}=\left[\pi_{2}(X)\right]^{\theta} \quad \text { a.e. }(\mu) \text { for all } \theta \notin M_{X} .
$$

We now define a stochastic process $\left\{Y_{\theta}\right\}_{\theta \in \Theta}$ by setting $Y_{\theta}=\varrho\left(X_{\theta}\right)$. It can be easily seen that $\left\{Y_{\theta}\right\}_{\theta \in \Theta}$ satisfies the required conditions. 
REMARK 5.3. Notice that in Theorem 5.2 we had to use properties of $\pi_{2}(X)$ in order to assure the plane measurability of $Y$. Directly defining $Y$ by $Y_{\theta}=\varrho\left(X_{\theta}\right)$ for an arbitrary lifting might destroy the plane measurability properties of the process $X$ (cf. [3]).

An important problem in the theory of functions of two variables is finding conditions guaranteeing the plane measurability of a separately measurable function. The notion of a stable set investigated by Fremlin and Talagrand (see [11]) turned out to be very fruitful in this field. In particular, Talagrand [11] (10-2-1) proved that if $\Theta$ is a compact set, $\nu$ is a Radon measure on $\Theta, f: \Omega \times \Theta \rightarrow \mathbb{R}$ is measurable as a function of the first variable and continuous as a function of the second variable, then $f$ is measurable provided the family $\left\{f^{\theta}: \theta \in \Theta\right\}$ is a stable set.

Applying a result of Fremlin [4] we get a similar result for functions that have lifting invariant sections. For the sake of completeness and because it is not published we present here Fremlin's result and his proof (with references to [11] rather than to Fremlin's notes).

Proposition 5.4. Let $(\Omega, \Sigma, \mu)$ and $(\Theta, T, \nu)$ be complete probability spaces. Let $f: \Omega \times \Theta \rightarrow \mathbb{R}$ be a separately measurable function such that $\left\{f^{\theta}: \theta \in \Theta\right\}$ is stable. Then there is a $\Sigma \widehat{\otimes} T$-measurable function $\widetilde{f}: \Omega \times \Theta \rightarrow \mathbb{R}$ such that $\tilde{f}^{\theta}=f^{\theta}$ a.e. $(\mu)$ for every $\theta$.

Proof. (a) Suppose first that $f$ is bounded and let $\mathcal{Z}$ be the pointwise closed convex hull of $\left\{f^{\theta}: \theta \in \Theta\right\}$. By [11] (11-2-1), $\mathcal{Z}$ is stable. Since $f$ is bounded the set $\mathcal{Z}$ is also pointwise compact. Furthermore, according to [11] (9-5-2), $\mathcal{Z}$ is also compact for the $L_{1}$-pseudometric. Thus, given $\varepsilon>0$ there are $L_{1}$-open sets $Z_{1}, \ldots, Z_{p} \subset \mathcal{Z}$ covering $\mathcal{Z}$ and such that $\int|g-h| d \mu \leq \varepsilon$ whenever $g, h$ belong to the same $Z_{i}$. Moreover, each $Z_{i}$ can be taken to be of the form $Z_{i}=\left\{h:\left|h(\omega)-\alpha_{\omega}^{i}\right| \leq \delta \forall \omega \in I_{i}\right\}$ for some finite $I_{i} \subset \Omega, \delta>0$ and $\left\langle\alpha_{\omega}^{i}\right\rangle_{\omega \in I_{i}} \in \mathbb{R}^{I_{i}}$.

Set $E_{j}:=\left\{\theta: f^{\theta} \in Z_{j}\right\}$. Then $E_{j}=\bigcap_{\omega \in I_{j}}\left\{\theta:\left|f_{\omega}(\theta)-\alpha_{\omega}^{j}\right| \leq \delta\right\} \in T$. Set $E_{j}^{\prime}:=E_{j} \backslash \bigcup_{i<j} E_{i}$ and write $J:=\left\{j: \nu\left(E_{j}^{\prime}\right)>0\right\}$ and $M=\Theta \backslash \bigcup_{j \in J} E_{j}^{\prime}$. Then, given $(\omega, \theta) \in \Omega \times E_{j}^{\prime}$ and $j \in J$, set

$$
g(\omega, \theta):=\frac{1}{\nu\left(E_{j}^{\prime}\right)} \int_{E_{j}^{\prime}} f(\omega, \xi) \nu(d \xi)
$$

and $g(\omega, \theta):=f(\omega, \theta)$ if $\theta \in M$. It is clear that $g$ is $\Sigma \widehat{\otimes} T$-measurable. Moreover, since $g^{\theta} \in Z_{j}$ if $\theta \in E_{j}^{\prime}$, we get $\int\left|g^{\theta}-f^{\theta}\right| d \mu \leq \varepsilon$ for every $\theta$.

(b) Let now $f$ be arbitrary. For each $n \in \mathbb{N}$ let $g_{n}$ be a $\Sigma \widehat{\otimes} T$-measurable function on $\Omega \times \Theta$ such that for each $n \in \mathbb{N}$ and $\theta \in \Theta$,

$$
\int\left|g_{n}^{\theta}-f_{n}^{\theta}\right| d \mu \leq 2^{-n}
$$


where $f_{n}(\omega, \theta):=\max \{-n, \min (n, f(\omega, \theta))\}$. One easily sees that $g_{n}^{\theta} \rightarrow f^{\theta}$ a.e. for every $\theta$ and that $\left\langle g_{n}\right\rangle_{n \in \mathbb{N}}$ is convergent a.e. to a $\Sigma \widehat{\otimes} T$-measurable function $\widetilde{f}$.

The following consequence of Proposition 5.4 and of the main result of this paper may be considered as a strengthening of Talagrand's method [12] of modification of a stochastic process with the help of consistent liftings.

Theorem 5.5. Let $(\Omega, \Sigma, \mu),(\Theta, T, \nu)$ be complete probability spaces and $\left\{X_{\theta}\right\}_{\theta \in \Theta}$ be an arbitrary real-valued bounded stochastic process on $(\Omega, \Sigma, \mu)$ with measurable paths (i.e. all functions $X_{\omega}$ are $\nu$-measurable). If the set $\left\{X_{\theta}: \theta \in \Theta\right\}$ is stable then for each $\varrho \in A G \Lambda(\mu)$ the process $\left\{Y_{\theta}\right\}_{\theta \in \Theta}$ given for each $\theta$ by $Y_{\theta}:=\varrho\left(X_{\theta}\right)$ is $\Sigma \widehat{\otimes} T$-measurable and equivalent to $\left\{X_{\theta}\right\}_{\theta \in \Theta}$.

Proof. Applying Proposition 5.4 we get a bounded $\Sigma \widehat{\otimes} T$-measurable function $\widetilde{X}: \Omega \times \Theta \rightarrow \mathbb{R}$ such that $\widetilde{X}^{\theta}=X_{\theta}$ a.e. $(\mu)$, for every $\theta$. If $\pi_{2} \in \Lambda(\mu \widehat{\otimes} \nu)$ is a lifting satisfying the assertion of Theorem $2.13^{\perp}$ (with $\sigma$ arbitrary), then $\pi_{2}(\widetilde{X})$ is measurable and for each $\theta$,

$$
\left[\pi_{2}(\widetilde{X})\right]^{\theta}=\varrho\left(\left[\pi_{2}(\tilde{X})\right]^{\theta}\right) .
$$

By the Fubini Theorem there is a set $M_{f} \in T_{0}$ such that $Y_{\theta}=\left[\pi_{2}(\widetilde{X})\right]_{\theta}$ for all $\theta \notin M_{f}$. It follows that $\left\{Y_{\theta}\right\}_{\theta \in \Theta}$ satisfies the required conditions.

Corollary 5.6. Let $(\Omega, \Sigma, \mu),(\Theta, T, \nu)$ be complete probability spaces and let $f: \Omega \times \Theta \rightarrow \mathbb{R}$ be a separately measurable function such that the set $\left\{f^{\theta}: \theta \in \Theta\right\}$ is stable. If there exists $\varrho \in A G \Lambda(\mu)$ such that $\varrho\left(f^{\theta}\right)=f^{\theta}$ for each $\theta \in \Theta$, then $f$ is $\Sigma \widehat{\otimes} T$-measurable.

\section{References}

[1] P. Billingsley, Probability and Measure, Wiley, New York, 1979.

[2] A. Blass, handwritten notes, 1999.

[3] D. L. Cohn, Liftings and the construction of stochastic processes, Trans. Amer. Math. Soc. 246 (1978), 429-438.

[4] D. H. Fremlin, Stable sets of measurable functions, Note of 17 May 1983.

[5] S. Graf and H. von Weizsäcker, On the existence of lower densities in noncomplete measure spaces, in: Measure Theory (Oberwolfach, 1975), A. Bellow and D. Kölzow (eds.), Lecture Notes in Math. 541, Springer, Berlin, 1976, 155-158.

[6] A. Ionescu and C. Tulcea, Topics in the Theory of Liftings, Springer, Berlin, 1969.

[7] G. Koumoullis, On perfect measures, Trans. Amer. Math. Soc., 264 (1981), 521-537.

[8] N. D. Macheras, K. Musiał and W. Strauss, On products of admissible liftings and densities, Z. Anal. Anwendungen 18 (1999), 651-667.

[9] N. D. Macheras and W. Strauss, On products of almost strong liftings, J. Austral. Math. Soc. Ser. A 60 (1996), 1-23. 
[10] W. Sierpiński, Fonctions additives non complètement additives et fonctions non mesurables, Fund. Math. 30 (1939), 96-99.

[11] M. Talagrand, Pettis Integral and Measure Theory, Mem. Amer. Math. Soc. 307 (1984).

[12] - On liftings and the regularization of stochastic processes, Probab. Theory Related Fields 78 (1988), 127-134.

[13] -, Closed convex hull of set of measurable functions, Riemann-measurable functions and measurability of translations, Ann. Inst. Fourier (Grenoble) 32 (1989), 39-69.

[14] -, Measurability problems for empirical processes, Ann. Probab. 15 (1987), $204-212$.

[15] T. Traynor, An elementary proof of the lifting theorem, Pacific J. Math. 53 (1974), $267-272$.

Institute of Mathematics

Wrocław University

Pl. Grunwaldzki 2/4

50-384 Wrocław, Poland

E-mail: musial@math.uni.wroc.pl

Department of Statistics

University of Piraeus

80 Karaoli and Dimitriou street

18534 Piraeus, Greece

E-mail: macheras@unipi.gr
Mathematisches Institut A

Universität Stuttgart

Postfach 801140

D-70511 Stuttgart, Germany

E-mail: strauss@mathematik.uni-stuttgart.de

Received 20 December 1999;

in revised form 10 May 2000 